\title{
Base Station Placement for Wireless Sensor Network Positioning System via Lexicographical Stratified Programming
}

\author{
Jun Yan ${ }^{1}$ and Kegen $\mathrm{Yu}^{2}$ \\ ${ }^{1}$ College of Telecommunications and Information Engineering \\ Nanjing University of Posts and Telecommunications, Nanjing China 210003 \\ [e-mail: yanj@njupt.edu.cn] \\ ${ }^{2}$ School of Geodesy and Geomatics and Collaborative Innovation Center for Geospatial Technology, \\ Wuhan University, Wuhan, China 430072 \\ [e-mail: kegen.yu@ieee.org] \\ *Corresponding author: Jun Yan
}

Received March 23, 2015; revised May 20, 2015; accepted September 2, 2015;

published November 30, 2015

\begin{abstract}
This paper investigates optimization-based base station (BS) placement. An optimization model is defined and the BS placement problem is transformed to a lexicographical stratified programming (LSP) model for a given trajectory, according to different accuracy requirements. The feasible region for BS deployment is obtained from the positioning system requirement, which is also solved with signal coverage problem in BS placement. The LSP mathematical model is formulated with the average geometric dilution of precision (GDOP) as the criterion. To achieve an optimization solution, a tolerant factor based complete stratified series approach and grid searching method are utilized to obtain the possible optimal BS placement. Because of the LSP model utilization, the proposed algorithm has wider application scenarios with different accuracy requirements over different trajectory segments. Simulation results demonstrate that the proposed algorithm has better BS placement result than existing approaches for a given trajectory.
\end{abstract}

Keywords: base station placement; Geometric dilution of precision (GDOP); Lexicographical Stratified Programming model; wireless sensor network positioning system

This research was supported by the National Natural Science Foundation of China (No. 61302103, 61372122, 61372123, 61170252) and Research Foundation for Advanced Talents of NJUPT (NY213012). 


\section{Introduction}

W ireless sensor network (WSN) has received significant attention from both academia and industry in the past decade [1]. Example applications include environmental monitoring, object tracking, surveillance and rescue operations. Positioning and tracking are the primary objective of a range of WSN-based applications and play a significant role in many other applications. Due to constraints on the cost and complexity of a WSN, the measurement accuracy of the typical off-the-shelf sensors can be low. Thus, how to improve the positioning performance of WSN-based positioning systems is a critical issue.

The overall positioning performance of a WSN-based positioning system depends on a number of factors including the accuracy of parameter (range, time of arrival, received signal strength, or heading angle) estimation, the choice of the positioning approach and the geometric relationship between the mobile station (MS) or mobile node and the base stations (BS) or anchor nodes [2]. That is, in a given WSN, the positioning performance will be affected by the positioning approach and the BS deployment. Therefore, development of innovative methods and techniques for obtaining appropriate BS placement is thus of great interest for WSN positioning system.

Some BS placement algorithms for positioning have been studied in the literature [3]-[13]. Since the Cramer-Rao bound (CRB) is usually considered as a benchmark for evaluating the accuracy of any unbiased location estimator, the authors of [3][4] studied how to optimize the BS placement to minimize the CRB for a given number of sensors and derived sufficient and necessary conditions for an unconstrained optimum sensor placement. It can be seen that for a given single point, the uniform angular array (UAA) pattern of BS placement is an optimal BS placement. In [5], the authors discussed a number of different BS placement strategies such as maximizing the Fisher information matrix, minimizing the Cramer-Rao bound, using the spherical codes (SC), and employing the UAA and platonic solids. They found that CRB-optimum sensor arrays are also FIM-optimum, but not vice versa. Meanwhile, the 2D SC approach is identical to the UAA method and they are more restrictive than the CRB-based approach. In [6], the authors proposed the necessary and sufficient conditions on the sensor-target geometry which leads to the minimization of the uncertainty ellipsic area of position estimation with an unbiased estimator when using range-only, bearing-only and time-of-arrival measurements respectively. Based on the results given in [6], the authors of [7] presented a unified framework to analyze the optimal sensor placements of bearing-only, range-only and received signal strength (RSS) measurements and also proved the necessary and sufficient conditions for optimal placements in 2D and 3D scenarios.

In [8], the author proposed a BS placement for a given area by minimizing the mean CRB. In the case of three anchors placed in a square area, the highest positioning accuracy is achieved when the trio is placed at the corners of an equilateral triangle. This triangle is of maximum size as two anchors are placed at the corners of one side of the square area while the third anchor is placed at the center of the opposite side. The best anchor locations for the case of four anchors are the four corners of the square area, while the best location for an additional fifth anchor is the center of the area. Some basic guidelines are provided in [9] [10] to minimize the error in location estimation. The authors observed that if the field of study is rectangular, the best BS configuration is a rectangular pattern and BSs are at the vertex based on geometric dilution of precision (GDOP) and multi-lateration rules. In [11], a BS placement strategy for physical measurements, which maximizes coverage and minimizes the positioning 
error, has been investigated in different scenarios. It is demonstrated that the optimal layout tends to be uniform placement of BS in the given area. In [12], uniform boundary placement, random boundary placement and random placement have been considered to evaluate the accuracy of the hop-count positioning system. It is shown that placing landmarks on the periphery of the topology yields more accurate coordinate estimation than deploying landmarks anywhere in the interior. Note that the above methods give a solution to BS placement in a given area, whereas the methods in [3]-[7] are limited to a single point.

In [13], a BS placement approach based on GDOP analysis was proposed for the medium range outdoor and indoor location system design. Different from the above mentioned methods, this approach focuses on the appropriate BS placement when giving a particular MS trajectory. In [14], it is mentioned that the optimal landmark deployment follows some simple and symmetric patterns and the stretching/shrinking may be used to reduce localization errors. However, it is also pointed out that there is a conflict between the ideal landmark deployment for localization and deployments for optimizing signal coverage.

From the above discussion and analysis, it can be seen that most existing BS placement strategies are based on a single point and a given area, little work has been done based on trajectories. With the advances of WSN technology, WSN positioning will be applied to many different applications. For the given trajectory, different positioning service may be corresponded to the different segments of the trajectory. Thus, the accuracy requirement of each segment can be different. For instance, in the case of horse racing, the position accuracy for the final track segment of the course should be higher. Another example is that near some specific landmarks the positions of the athletes need to be tracked precisely so that the athletes' performance can be compared with each other or with the record. Accordingly, the BS placement design should put more weight on the segment of higher accuracy requirement. Also, the probability of MS on each point of the given trajectory can also be different. The points with higher probability play more important role in the BS placement design. Thus, for a given trajectory, obtaining different weights for different segments poses new challenges to BS placement.

Motivated by the above observation, in this paper, we focus on studying BS placement for a given but not specific trajectory with different segment accuracy requirement in a complex environment. In particular, a simple and effective BS placement approach is proposed. Taking the average GDOP as a performance measure, the placement problem is formulated as a mathematical optimization modeling issue. To produce a unique position estimate, the number of the BS or the received signal must be greater than the dimensions of location by at least one. Meanwhile, from the viewpoint of data processing, larger number of the received signal can improve the positioning performance. Thus, utilizing this knowledge, the feasible region of the optimization model is determined with the given number of received BS signal. Through this processing, the problem of signal coverage in BS placement is first resolved. In order to cope with different accuracy requirement, the optimization model is further transformed to a lexicographical stratified programming (LSP) model using the priority parameters related to accuracy. Under different accuracy requirements, the given trajectory is divided into several segments. The average GDOP of each segment is chosen as the objective function. Based on the objective functions and feasible region, a tolerant factor based complete stratified series (TFBCSS) approach and a grid searching approach are utilized to generate the optimal solution to the BS placement.

The remainder of this paper is organized as follows. Section 2 describes the proposed BS placement algorithm in detail. Section 3 presents the algorithm analysis. Section 4 tells about the simulation design and shows the simulation results. Section 5 draws our conclusions. 


\section{Proposed algorithm}

\subsection{Some assumptions}

For the sake of simplicity, the following assumptions are taken into consideration.

(1) 2D positioning is assumed and without loss of generality, the given region is rectangular and divided into $M \times N$ grids which are the potential sites for BS placement [9].

(2) The coverage or radio range of each BS is limited to a fixed circular area with radius R.

(3) The trajectory is divided into several segments which may have different position accuracy requirements. A certain number of position points are extracted from each trajectory segment, depending on the sampling frequency and the MS speed.

\subsection{Basic idea of the algorithm}

Fig. 1 shows the block diagram of the proposed BS placement method. In the mathematical optimization model, the position coordinates of the BSs are the variables, while the average GDOP of each trajectory segment is defined as the objective function. The BS placement problem is first formulated as a multiple objective optimization model. Then, based on the different accuracy requirement of each segment, this model is further transformed to a LSP model by the priority parameters. At last, a TFBCSS approach and a grid searching approach are adopted to produce the optimization solution and realize the optimal BS placement. In the following section, the proposed algorithm is described in detail.

Note that the GDOP-based and CRLB-based criteria for BS placement design are identical, when the measurement noise is modelled as a Gaussian random variable [15] [16].

\subsection{Multiple objective optimizations}

Let $\left(x_{i}, y_{i}\right)$ be the position of the $\mathrm{i}^{\text {th }} \mathrm{BS}$ and there are $\mathrm{n}$ BSs to be deployed. The $\mathrm{m}^{\text {th }} \mathrm{MS}$ position coordinates are denoted as $\left(a_{m}, b_{m}\right)$ and there are M MS position samples on the whole MS trajectory. Denote $f_{j}\left(x_{1}, y_{1}, x_{2}, y_{2}, \cdots x_{n}, y_{n}\right)$ as the average of the GDOPs at the MS position points on the $\mathrm{j}^{\text {th }}$ segment and there are totally $\mathrm{L}$ trajectory segments. The average GDOP is a function of the positions of the BSs and MS. More details about GDOP calculation can be found in [13] [17]. For simplicity, define

$$
X \stackrel{\Delta}{=}\left(x_{1}, x_{2}, \cdots, x_{n}\right), \quad \stackrel{\Delta}{=}\left(y_{1}, y_{2}, \cdots, y_{n}\right)
$$

Then, the objective function is defined as

$$
G(X, Y) \stackrel{\Delta}{=}\left(f_{1}(X, Y), \cdots, f_{L}(X, Y)\right)^{T}
$$




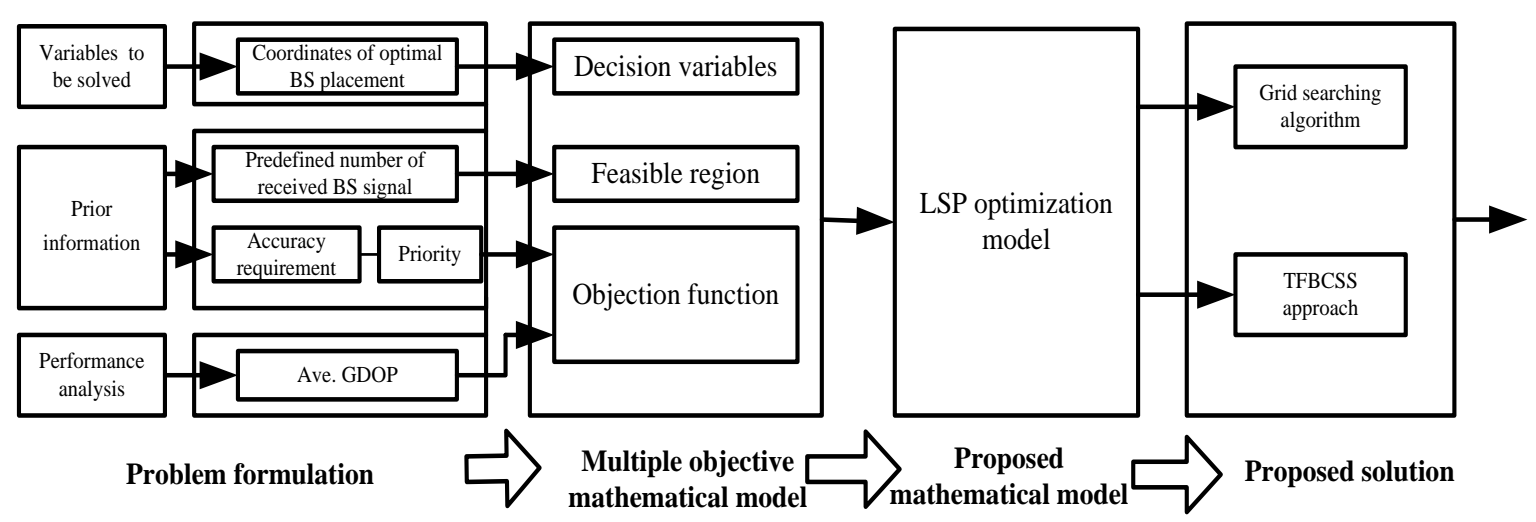

Fig. 1. Block diagram of the proposed BS placement scheme

The multiple objective optimizations for BS placement can be defined as:

$$
(X, Y)=\min _{X, Y} f_{j}(X, Y), \quad j=1,2, \cdots, L
$$

Assume that there are at least N BSs which are within the radio range of the MS at each position point on the trajectory. Mathematically, feasible regions are denoted as

$$
D(X, Y) \stackrel{\Delta}{=}\left\{(X, Y) \mid g_{m}(X, Y) \geq N \quad m=1, \cdots M\right\}
$$

where $g_{m}(X, Y)=\sum_{i=1}^{n} \varepsilon\left(\sqrt{\left(x_{i}-a_{m}\right)^{2}+\left(y_{i}-b_{m}\right)^{2}}-R\right), \varepsilon(t)$ is a unit step function and is defined as $\varepsilon(t)= \begin{cases}1 & t \geq 0 \\ 0 & t<0\end{cases}$

\subsection{LSP model for BS placement}

In order to accommodate the different accuracy requirement for each segment of the trajectory, in the next, the multiple objective optimization models are transformed to a LSP model. Based on the predefined priority of each segment with respect to the accuracy requirement, in the following, the transformation is described in details.

Assume that there are $k$ different accuracy requirements of the MS trajectory. Hence, the L objective functions can be represented as:

$$
\begin{gathered}
f_{1}^{1}(X, Y), \cdots f_{L_{1}}^{1}(X, Y) ; f_{1}^{2}(X, Y), \cdots f_{L_{2}}^{2}(X, Y) ; \ldots \ldots f_{1}^{k}(X, Y), \cdots f_{L_{k}}^{k}(X, Y) ; \\
L_{1}+L_{2}+\cdots+L_{k}=L
\end{gathered}
$$

Where the $1^{\text {st }}$ priority level is $f_{1}^{1}(X, Y), \cdots f_{L_{1}}^{1}(X, Y)$, the $2^{\text {nd }}$ priority level is $f_{1}^{2}(X, Y), \cdots f_{L_{2}}^{2}(X, Y), \ldots \ldots \ldots$, the $\mathrm{k}^{\text {th }}$ priority is defined as $f_{1}^{k}(X, Y), \cdots f_{L_{k}}^{k}(X, Y)$. 
According to the discussion above, the problem of stratified multiple objective minimization can be defined as

$$
L-\min _{(X, Y) \in D}\left[P_{1}\left(f_{1}^{1}(X, Y), \cdots f_{L_{1}}^{1}(X, Y)\right), \cdots P_{k}\left(f_{1}^{k}(X, Y), \cdots f_{L_{k}}^{k}\right)\right]
$$

Where $P_{s}(s=1,2 \cdots k)$ is the priority sign and describes the objective functions in the bracket belong to the $\mathrm{s}^{\text {th }}$ priority. Meanwhile $P_{s} \gg P_{s+1}, s=1, \cdots k$, the sign " $>$ " is only the qualitative concept to indicate the $\mathrm{s}^{\text {th }}$ priority is higher than $(\mathrm{s}+1)^{\text {th }}$ priority. The sign " $\mathrm{L}-\mathrm{min}$ " indicates the lexicograhhical order minimization. It means that the optimization model is minimized from $P_{1}$ to $P_{k}$.

Define $F_{1}(X, Y)=\left(f_{1}^{1}(X, Y), \cdots f_{L_{1}}^{1}(X, Y)\right), F_{2}(X, Y)=\left(f_{1}^{2}(X, Y), \cdots f_{L_{2}}^{2}(X, Y)\right), \ldots$, $F_{k}(X, Y)=\left(f_{1}^{k}(X, Y), \cdots f_{L_{k}}^{k}(X, Y)\right)$,

Therefore, the above optimization problem can be rewritten as

$$
L-\min _{(X, Y) \in D}\left[P_{s} F_{s}(X, Y)\right]_{s=1}^{k}
$$

Note that the equation (5) is the proposed LSP model for BS placement.

\subsection{Solution for LSP model}

In order to avoid the problem of a unique solution in middle priority level and reduce computational complexity, the TFCSS approach in [18] [19] and the grid searching method are utilized to solve the LSP optimization problem. The basic idea is that according to the predefined priority, the optimal solving set of each objective function is obtained in turn. Then the optimal result for the last priority level-objective function is the final solution. The following steps provide more details about the LSP optimization procedure.

Step 1: Initialization

$$
D^{1}(X, Y)=D(X, Y), s=1
$$

where $D(X, Y)$ is the initial feasible region of the multiple objective model.

Step 2: According to the different accuracy requirements, the segments with the same accuracy requirement are considered to have the same priority for minimization. The higher the accuracy requirement is, the higher the priority for minimization is.

In the same priority, the minimization calculation $F_{s}(X, Y)(s=1,2, \cdots k)$ can be formulated to be a single objective function optimization by the linear-based weight method. Thus, the objective function $Q_{q}(X, Y)(q=1,2, \cdots k)$ for different priority minimization in LSP model is defined as:

$$
Q_{q}(X, Y)=w_{1} f_{1}^{q}(X, Y)+\cdots+w_{L_{q}} f_{L_{q}}^{q}(X, Y) q=1, \cdots k \quad w_{1}+w_{2}+\cdots+w_{L_{q}}=1
$$

In the paper, for simplicity, the weight $w_{i}$ for each objective function in the same priority are identical. 
Step 3: Performing minimization according to the lexicograhical order.

$$
\min _{(X, Y) \in D^{s}} Q_{s}(X, Y)
$$

Using the grid searching approach, the optimal solution $\left(X^{s}, Y^{s}\right)$ and the optimal value of the objective function $Q_{s}\left(X^{s}, Y^{s}\right)$ are obtained.

Step 4: Test the number of stratification

(1) If $s=k$, then output the BS placement result: $(X, Y)=\left(X^{s}, Y^{s}\right)$

(2)If $s<k$, then go to Step 5

Step 5: Build the feasible region of the following stratification

$$
D^{s+1}=\left\{(X, Y) \in D^{s} \mid Q_{s}(X, Y) \leq Q_{s}\left(X^{s}, Y^{s}\right)+\delta_{k}\right\}
$$

Where $\delta_{k}$ is the tolerant factor. $s=s+1$, go to Step 3 .

\subsection{Summary}

In summary, the proposed approach consists of the following steps:

1) Discretize the given area; Sample the MS trajectory and obtain the approximate representation of the given trajectory; Based on the different accuracy requirements, divide the trajectory into corresponding segments.

2) According to the predefined received BS signals, obtain the feasible region of the optimization model by the samples of the trajectory and the coverage radius.

3) Take average of GDOP of each segment as the objective function, the multiple objective optimization model is formed.

4) By considering the accuracy requirements, the optimization model is further formulated as a LSP model.

5) By utilizing the tolerant factor based complete stratified series approach and the searching method, the optimal BS placement can be obtained by solving the LSP model.

Note that when the trajectory is under the same priority, the proposed algorithm is equivalent to the algorithm presented in [13] where analytical results with same priority are provided. Analytical results related to a trajectory with different priorities may be derived in a similar way and this would be interesting future research work.

\section{Algorithm analysis and comparison}

In order to evaluate the performance of the proposed BS placement algorithm, the prior works of [6] [7] [8] [13] are chosen for algorithm comparison.

(1) The main contribution of this paper is that the problem of BS placement is modeled as a LSP optimization issue, according to the different accuracy requirements. Thus, it has broader application scenarios, compared with the method for the given trajectory in [13].

(2) Different from the works of [13] where the authors only considered a specific trajectory for BS placement, in this paper, a universal BS placement approach was proposed. Meanwhile the proposed algorithm considers the signal coverage as a factor affecting the placement solution. 
In the proposed algorithm, the effect of signal coverage is first presented and utilized to obtain the feasible region of the optimization model. Thus, the proposed algorithm is more suitable for practical applications.

(3) Although both the proposed algorithm and the method in [13] adopt the GDOP as a BS placement criterion, they are rather different, because the proposed algorithm is able to cope with any type of trajectory, instead of a particular one. Furthermore, in this paper, a tolerant factor which can lead all the objective function of different priority to have effect on the BS placement and the grid searching approach which avoids the problem of local minimum value ensure the performance of the proposed approach.

(4) It is noticed that the existing BS placement strategies are developed when a single point or an area is given [6]-[8]. On the other hand, the proposed algorithm is derived when giving a specific trajectory. As a consequence, it is rather difficult to undertake comparative simulation studies because of the different application scenarios for BS placement.

\section{Simulation results}

Simulation results are provided to assess the performance of the proposed BS placement method in different application scenarios. Four BSs are assumed to be available for all simulation environments. The trajectory of the MS and the given areas for simulation are shown in Fig. 2. The trajectory contains three different segments (namely Load 1, Load 2 and Load 3). The Load 1, Load 2 and Load 3 are from A to B, C to D and E to F respectively. The sampling interval of each load is $20 \mathrm{~m}$ so that 11 samples are obtained over each load. The coordinates of $\mathrm{X}$-axis and $\mathrm{Y}$-axis for the given area are both limited within [0m, 2000m]. The dimensions of each grid along the $\mathrm{X}$-axis and $\mathrm{Y}$-axis are both $200 \mathrm{~m}$. The potential coordinates of BS are assumed to be $(200(i-1) m, 200(j-1) m$ ) (for $i, j=1, \cdots 11$ ). The coverage radius of each BS is $1200 \mathrm{~m}$. Although more measurements can be used to improve accuracy, the predefined number of received BS signals in this paper is 4, accounting for some realistic scenarios. The accuracy requirement of Load1, Load2 and Load3 are high, medium and low, respectively. The tolerant factor which can relax the feasible region of the next stratified minimization is set at $\delta_{k}=0.1$. For performance comparison, the algorithm which does not employ priority strategy [13] (No Priority) and the method which only considers the smallest average GDOP of each load in the feasible region (Theory) are selected.

\subsection{Performance of the signal coverage and GDOP}

First, the signal coverage or radio range of the proposed BS placement algorithm is shown in Fig. 3. From the figure, it can be seen that each position of the given trajectory can receive 4 BS signals, which is suitable for the predefined condition. Then, the GDOP values are depicted in Fig. 4. In the figure, because Load 1 has the highest priority, the GDOP is closer to the theoretical value than that with no priority. Since the smaller the GDOP is, the better the performance of positioning system is, it can be concluded that the proposed BS placement can be utilized to satisfy different accuracy requirements for segments of a given trajectory. 


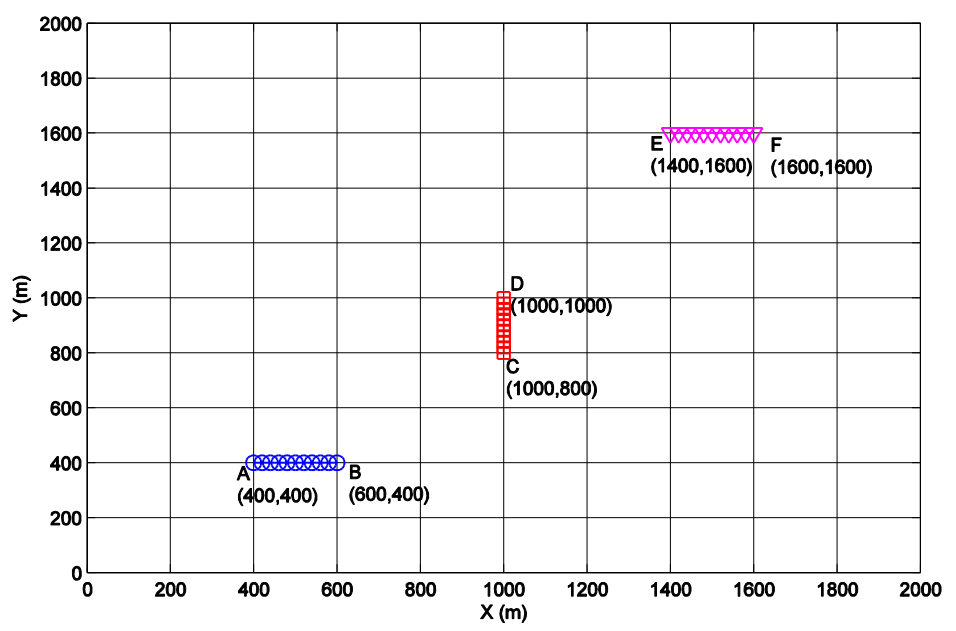

Fig. 2. Trajectory of the BS and the given areas for simulation

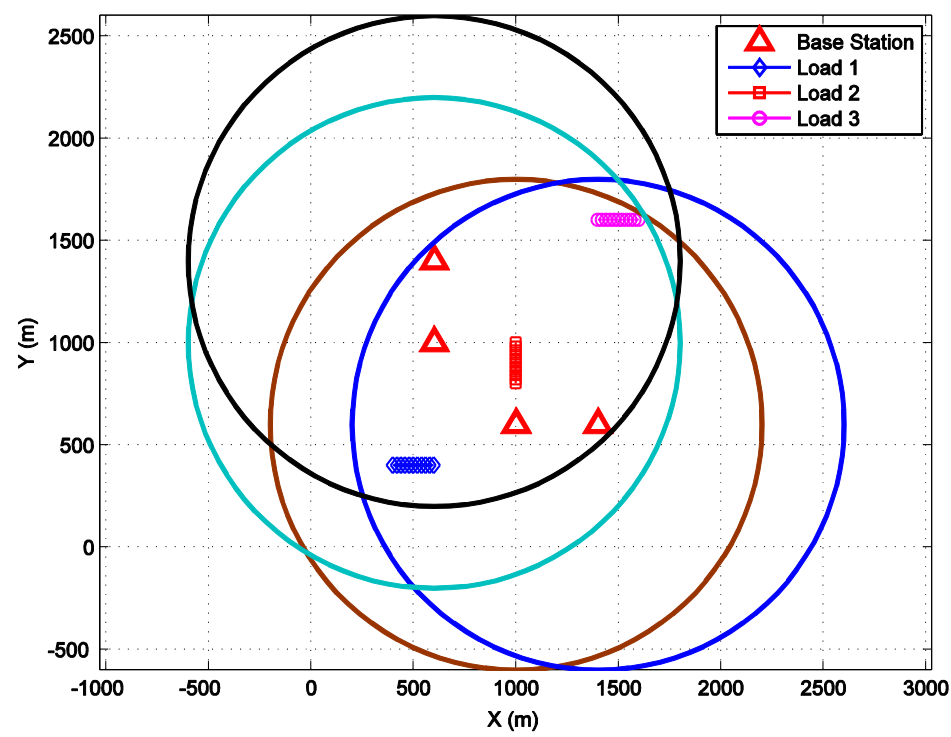

Fig. 3. The signal coverage or radio range of the BSs

\subsection{Robust test}

First, the robustness of the proposed algorithm with respect to different accuracy requirement is described. The above mentioned accuracy requirement is denoted as case 1 . In the following, the simulation condition where the accuracy requirement of Load1 and Load2 and load 3 are medium, low and high is denoted as case 2. Fig. 5 shows the signal coverage of the BSs, while Fig. 6 illustrates the GDOP of the two cases. From the figure, it can be seen that the obtained GDOP is closer to the theoretical value, when the priority of the segment is higher. Thus, the proposed algorithm is robust to different accuracy requirements.

Then, the robustness of the proposed algorithm with respect to the tolerant factor is evaluated for case 1. As shown in Fig. 7 and Fig. 8, the signal coverage and the GDOP are examined when the tolerant factor is set to be two different values: 0.1 and 0.15 . It can be seen that the tolerant factor does not have any effect on the signal coverage. The reason can be 
attributed to the fact that the problem of signal coverage is solved prior to obtaining the GDOP optimization solution and is not utilized for objective function construction. Meanwhile it can be observed that as the tolerant factor decreases, the GDOP with higher priority is close to the corresponding theoretical value (Load 1). However, the GDOP with lower priority is far away from the corresponding theoretical value ( $\operatorname{Load} 2$ and 3 ). Because of the smaller tolerant factor, the feasible region of the each stratified objective function becomes smaller and some minimum value in the next stratification will be lost. Hence, the tolerant factor selection is important for BS placement.

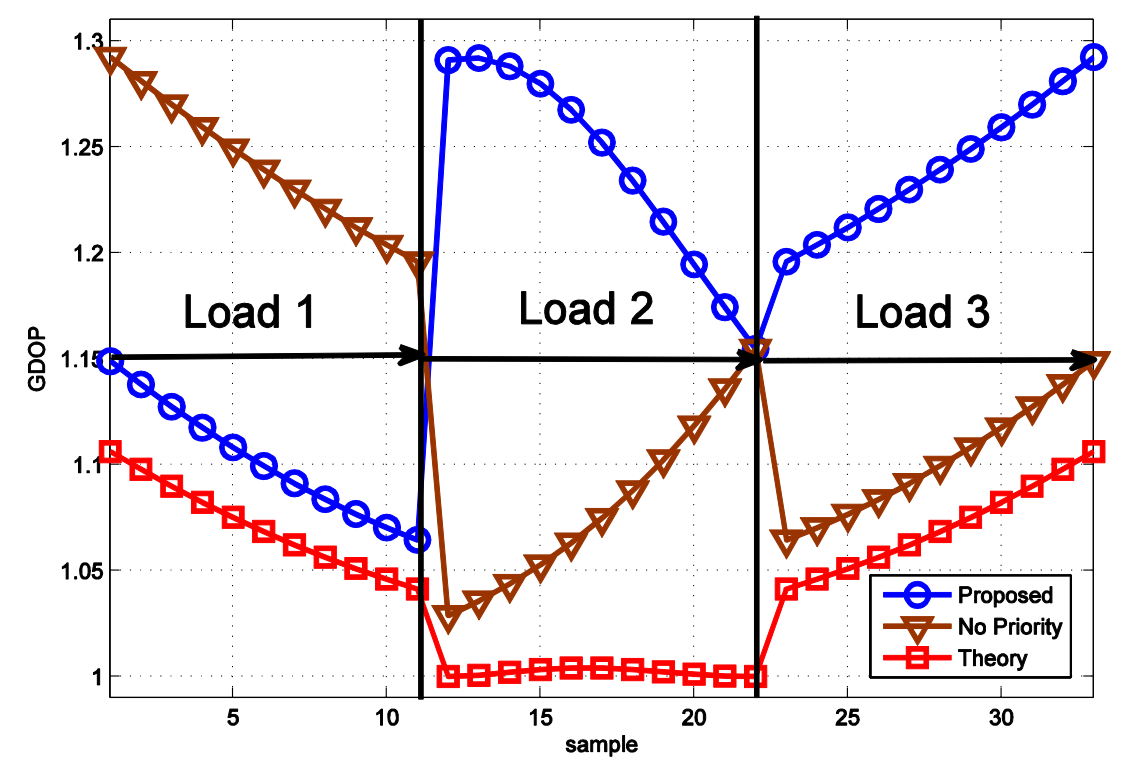

Fig. 4. GDOP description of the proposed algorithm and two other methods

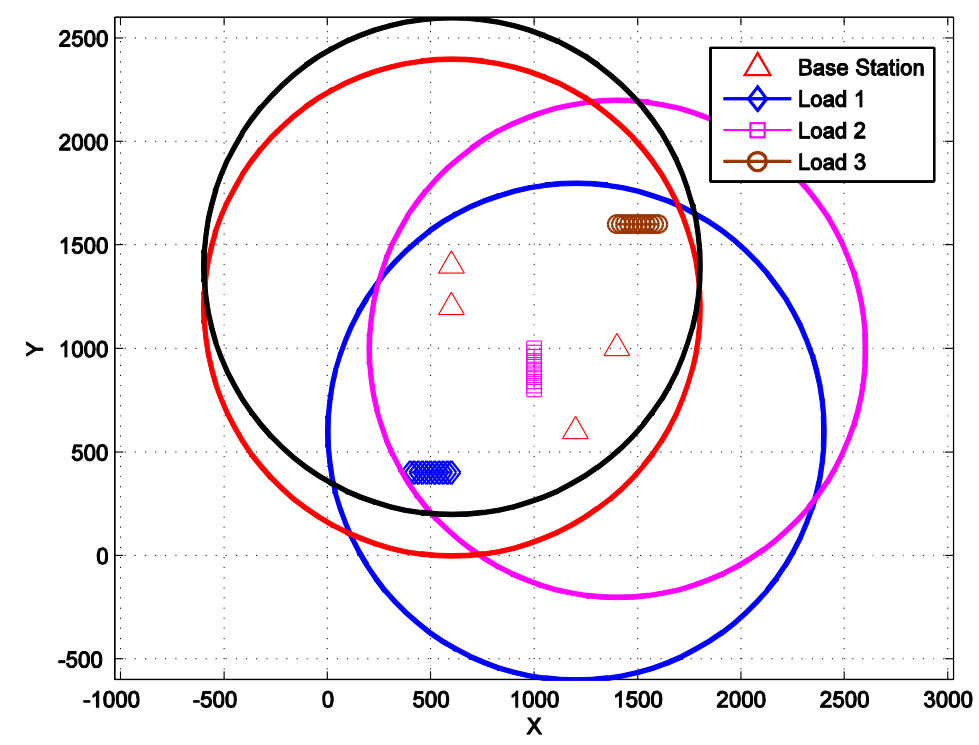

Fig. 5. Impact of accuracy requirement on signal coverage 


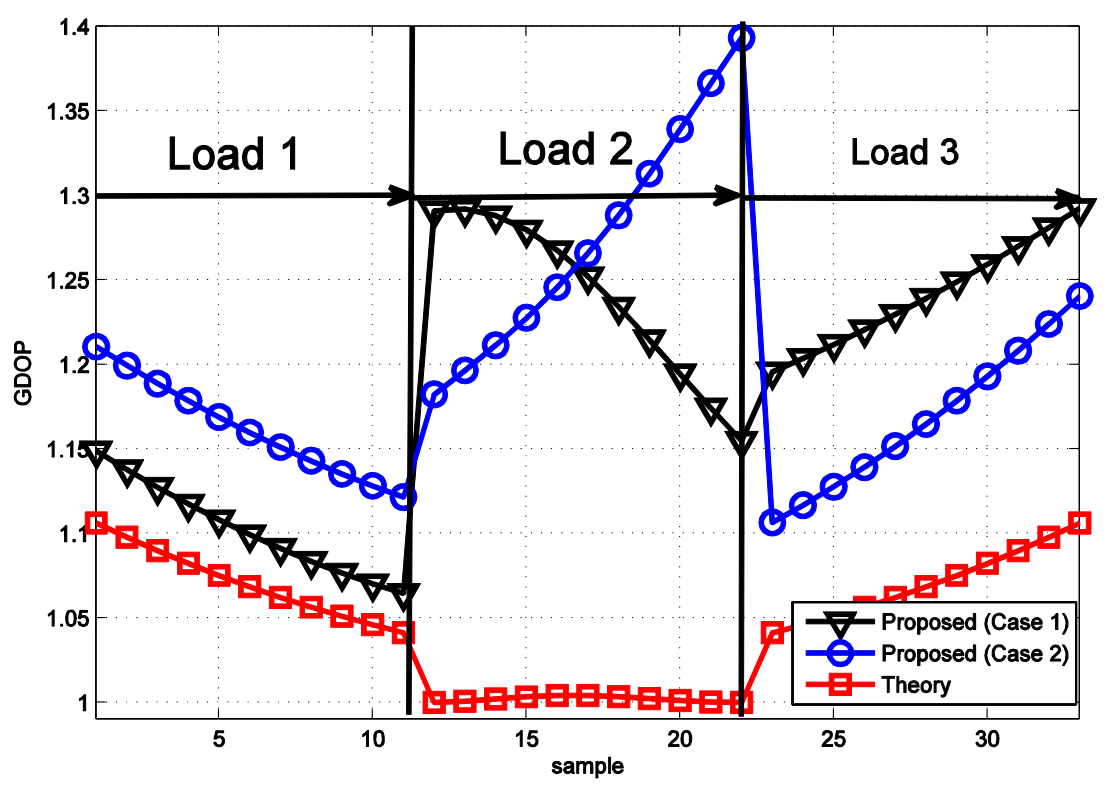

Fig. 6. Impact of accuracy requirement on GDOP

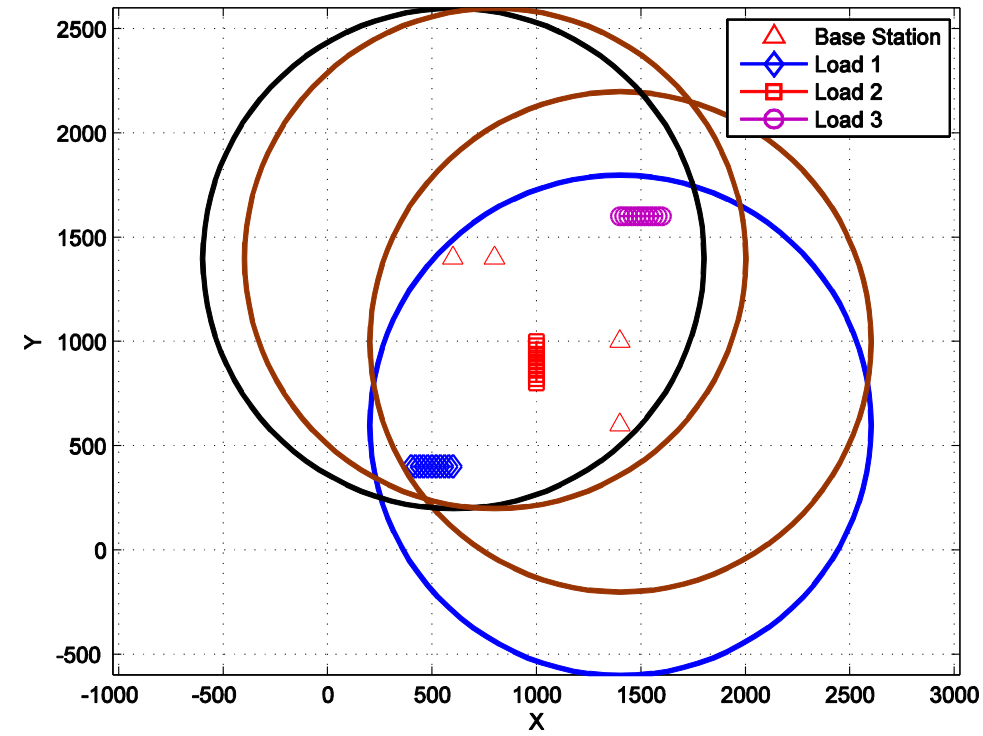

Fig. 7. Impact of tolerant factor on signal coverage

Next, the robustness of the proposed algorithm with respect to the sample ratio of the MS trajectory is analyzed for case 1 . In the robust test, a sampling interval of $30 \mathrm{~m}$ is chosen for performance comparison. From Fig. 9, it can be readily seen that the right hand side of Load3 does not receive signals from all four BSs. Thus, in this condition, the design requirement is not satisfied. Therefore, efficient sampling of the trajectory should be ensured for the BS placement. It can be concluded that BS placement is sensitive to sampling interval of the trajectory. 


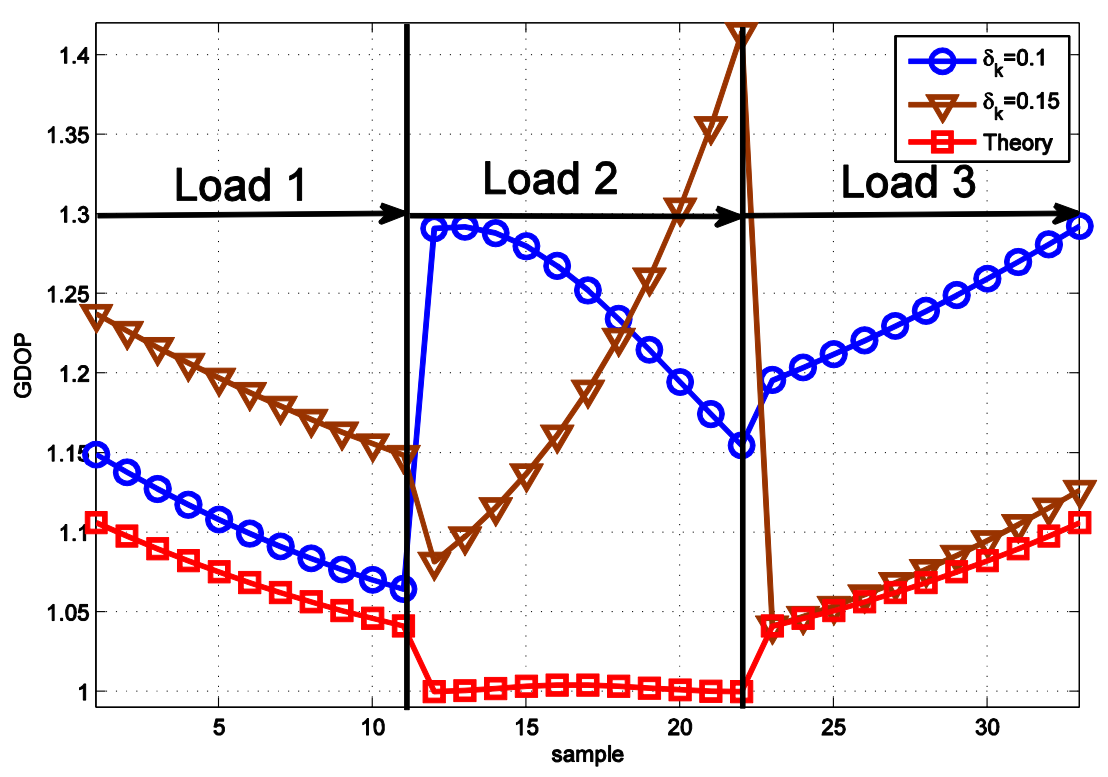

Fig. 8. Impact of tolerant factor on GDOP

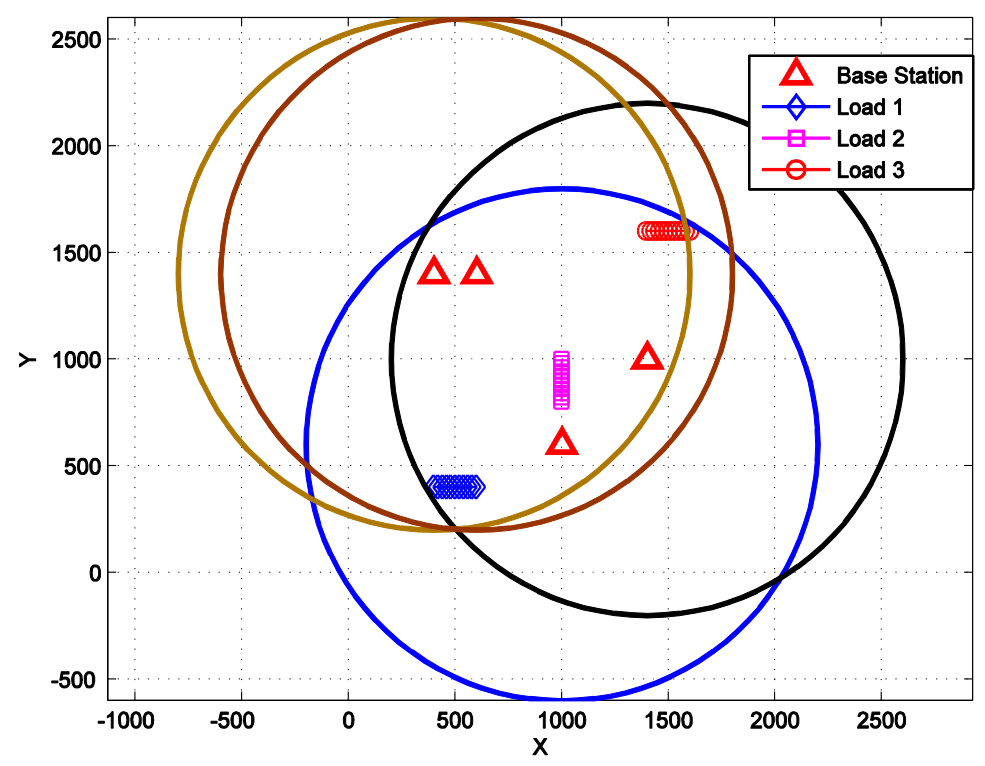

Fig. 9. Impact of sampling interval of trajectory on signal coverage

At last, different sampling interval of $\mathrm{X}$-axis and $\mathrm{Y}$-axis are chosen to test the performance of the proposed algorithm. In the robust test, only the sampling interval of Y-axis is increased by $100 \%$. Thus, the potential coordinates of BS become $(200(i-1) m, 400(j-1) m$ ) (for $i=1,2, \cdots 11, j=1,2, \cdots 6$ ). Fig. 10 and Fig. 11 show the performance in terms of signal coverage and average GDOP. From the figures, it can be concluded that larger potential coordinates of BSs can lead to higher GDOP but the signal coverage is not affected. 


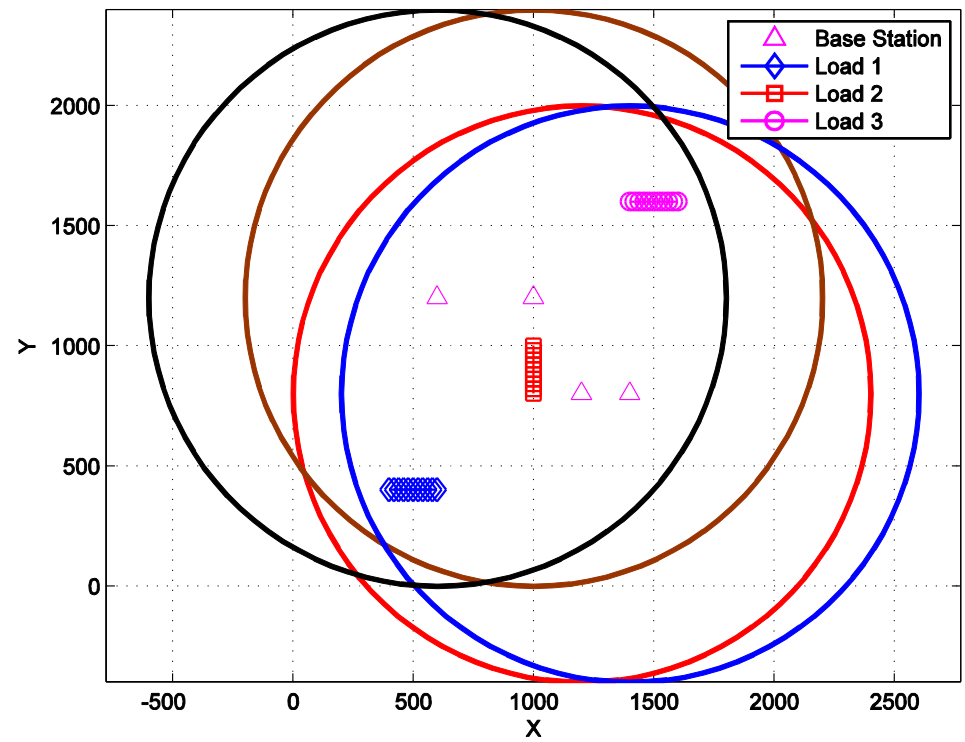

Fig. 10. Impact of sampling interval on signal coverage

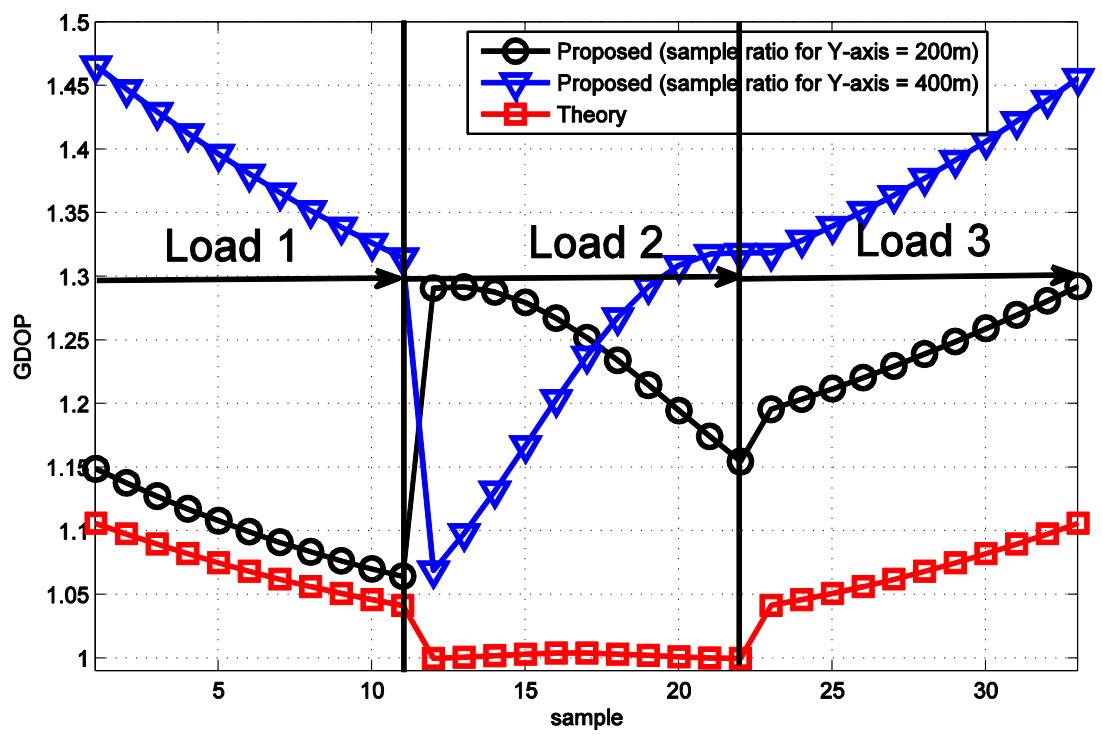

Fig. 11. Impact of sampling interval on GDOP

\subsection{Positioning performance analysis}

At last, the location performance (RMSE) of the proposed BS placement algorithm is compared with the optimization based method. In this simulation, the measurement noise is assumed to have a Gaussian distribution. Taking Load 1 and Case 1 as an example, Fig. 12 illustrates the RMSE comparison between the proposed BS placement algorithm and the no-priority BS placement approach, when the standard deviation of measurement noise is $10 \mathrm{~m}$. From the figures, it can be seen that the RMSE of the proposed BS placement method is smaller than that of the no priority method obviously. For another, the CRLB [20] of the 
proposed algorithm is smaller than that of the no priority method. Fig. 13 shows the average RMSE with different standard deviation of the measurement noise associated with Load 1. From the figure, it can be seen that the proposed BS placement approach has better location result than the no priority method. Meanwhile, the location performance of the proposed BS placement approach is closer to the theoretical BS placement strategy for Load 1. Thus, it can be concluded that the proposed algorithm can obtain better location performance when the accuracy requirements is high.

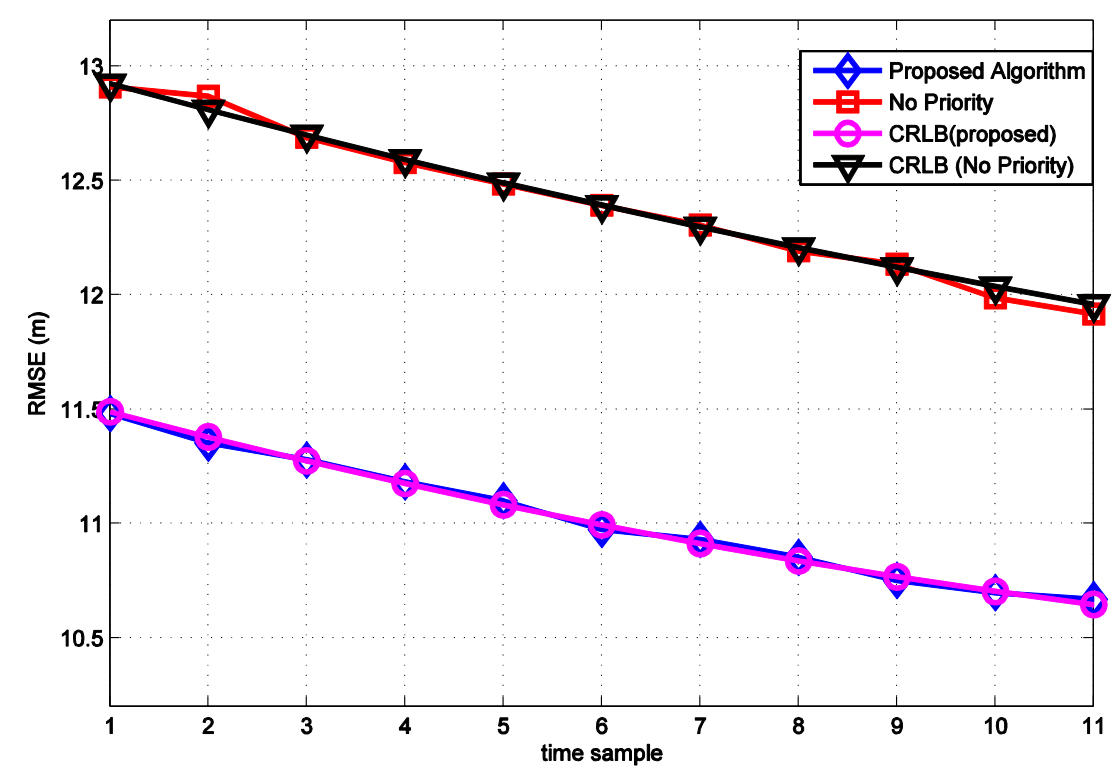

Fig. 12. RMSE comparison of different BS placement approach

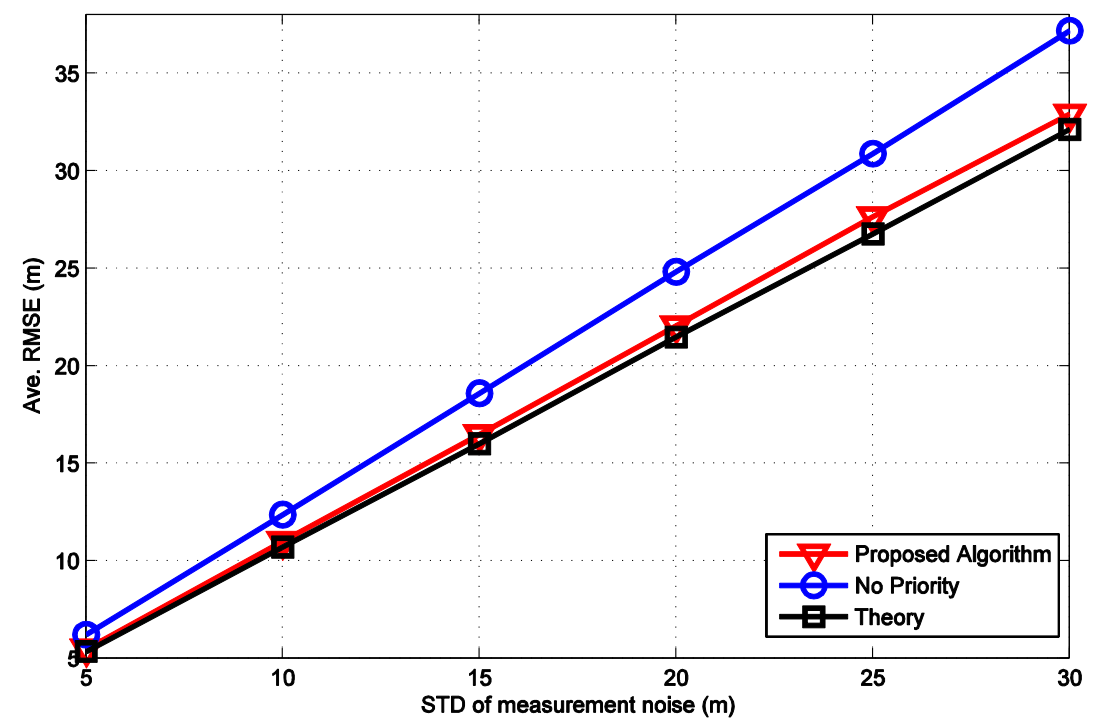

Fig. 13. Average RMSE comparison of different BS placement approach 


\section{Conclusions}

In this paper, a new BS placement algorithm has been presented for scenarios where there are different accuracy requirements. From the viewpoint of the positioning system, the problem of signal coverage is first dealt with. Based on the accuracy requirement, the BS placement problem is formulated as the LSP optimization model. A tolerant factor based complete stratified series approach which lead all the objective functions of different priority to have effect on the BS placement design and the grid searching approach which can avoid the problem of local minimum value are utilized to obtain the BS placement solution. Because of the LSP model utilization, the proposed algorithm has broader application scenarios and more suitable for practical applications. Simulation results demonstrate that the proposed algorithm performs better than previous work when a mobile trajectory is given.

\section{References}

[1] L. F. Akyildiz, W. Su, Y. Sankarasubramaniam, E. Cayirci, “A survey on sensor networks," IEEE Communications Magazine, vol. 4, no.8, pp. 102-114, Aug. 2002. Article (CrossRef Link)

[2] I. Sharp, K. Yu, M. Hedley, “On the GDOP and accuracy for indoor positioning," IEEE Trans. Aerospace and Electronic Systems, vol. 48, no.3, pp. 2032-2051, July 2012. Article (CrossRef Link).

[3] B. Yang, J. Scheuing, "Cramer-rao bound and optimum sensor array for source localization from time differences of arrival," ICASSP, IV pp. 961-964, 2005. Article (CrossRef Link).

[4] B. Yang, J. Scheuing, "A theoretical analysis of 2D sensor arrays for TDOA based localization,” ICASSP, IV pp. 901-904, 2006. Article (CrossRef Link).

[5] B. Yang, "Different sensor placement strategies for TDOA based localization," ICASSP, II 1093-1096, 2007. Article (CrossRef Link).

[6] A. N. Bishop, B. Fidan, B. D. O. Anderson, K. Dogancay, and P. N. Pathirana, "Optimality analysis of sensor-target localization geometries," Automatica, vol. 46, pp. 479-492, 2010. Article (CrossRef Link).

[7] S. Zhao, B. M. Chen, and T. H. Lee, "Optimal sensor placement for target localization and tracking in 2D and 3D," International Journal of Control, vol. 86, no. 10, pp. 1687-1704, 2013. Article (CrossRef Link).

[8] N. Salman, H. K. Maheshwari, A. H. Kemp, M. Ghogho, "Effects of anchor placement on mean-CRB for localization," in Proc. of $10^{\text {th }}$ IFIP Annual Mediterranean Ad Hoc Networking Workshop, pp.115-118, 2011. Article (CrossRef Link).

[9] Y. Chen, J. A. Francisco, W. Trapper, R. Martin, "A practical approach to landmark deployment for indoor localization," in Proc. of Third Annual IEEE Communications Society Conference on Sensor and AdHoc Communications and Networks, vol. 1, pp. 365-373, Sept. 2006. Article (CrossRef Link).

[10] J. Zhou, J. Shi, X. Qu, "Landmark placement for wireless localization in rectangular-shaped industrial facilities," IEEE Trans. Vehicular Technology, vol. 59, no. 6, 3081-3090, Jul. 2010. Article (CrossRef Link).

[11] A. Bais, Y. Morgan, "Evaluation of base station placement acenarios for mobile node localization," in Proc. of Third FTRA International Conference on Mobile Ubiquitous and Intelligent Computing, 201-206, 2012. Article (CrossRef Link).

[12] F. Benbadis, K. Obraczka, J. Cortes, A. Brandwajn, "Exploring landmark placement strategies for self-localization in wireless sensor networks," in Proc. of the 18th Annual IEEE International Symposium on Personal, Indoor and Mobile Radio Communications (PIMRC'07) Article (CrossRef Link).

[13] I. Sharp, K. Yu, Y. J. Guo, "GDOP analysis for positioning system design," IEEE Trans. Vehicular Technology, vol. 58, no. 7, pp. 3371-3382. Article (CrossRef Link). 
[14] Y. Chen, J.-A. Francisco, W. Trappe, R. P. Martin, “A practical approach to landmark deployment for indoor localization," IEEE Communications Society SECON, 365-373, 2006. Article (CrossRef Link).

[15] C. H. Chen, K. T. Feng, C. L. Chen, P. H. Tseng, "Wireless location estimation with the assistance of virtual base stations," IEEE Trans. Vehicular Technology, vol.58, no.1, pp.93-106, 2009. Article (CrossRef Link).

[16] J. Chaffee, J. Abel. "GDOP and the Cramer-Rao bound," in Proc. IEEE PLANS, Las Vegas, NV, pp. 663-668, 1994. Article (CrossRef Link).

[17] N. Levanon, "Lowest GDOP in 2-D scenarios," IEE Proc-Radar, Sonar, Navigation, vol.147, no. 3, pp. 149-155, 2000. Article (CrossRef Link).

[18] Y. Sawaragi, H. Nakayama, T. Tanino, "Theory of multiobjective potimization, Mathematics in Science and Engineering," vol. 176, Academic Press, 1985.

[19] S. M. Lee, "Goal programming for decision analysis," Auerbach Publishers Inc., Philadelphia, 1972.

[20] S. Wu, J. Li , S. Liu, "Improved Localization Algorithms Based on Reference Selection of Linear Least Squares in LOS and NLOS Environments," Wireless Pers. Commun, Vol.68, pp. 187-200, March, 2013. Article (CrossRef Link).
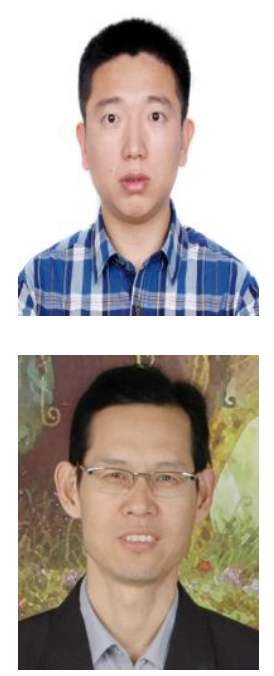

Jun Yan received his Ph.D degree in electrical engineering from Southeast University, Nanjing, China in 2012. He is currently a assistant professor at College of Telecommunications and Information Engineering, Nanjing University of Posts and Telecommunications. His research interest is statistical signal processing for wireless location.

Kegen $Y \mathbf{u}$ received the Ph.D. degree in electrical engineering from the University of Sydney, Sydney, NSW, Australia, in 2003. He is currently a Professor with the School of Geodesy and Geomatics, Wuhan University, Wuhan, China. He has worked for Jiangxi Geological and Mineral Bureau, Nanchang, China, Department of Electrical Engineering at Nanchang University, CWC at the University of Oulu, CSIRO ICT Centre, Department of Electronic Engineering at Macquarie University, and School of Surveying and Geospatial Engineering (now integrated within the School of Civil and Environmental Engineering) and the Australian Centre for Space Engineering Research within the University of New South Wales. He has been an Adjunct Professor with Macquarie University since 2011. Dr. Yu is currently on the editorial boards of the EURASIP Journal on Advances in Signal Processing, the IEEE Transactions on Aerospace and Electronic Systems, and the IEEE Transactions on Vehicular Technology. He is the Lead Guest Editor for a Special Issue of Physical Communication on Navigation and Tracking and for a Special Issue of the EURASIP Journal on Advances in Signal Processing on GNSS Remote Sensing. 